\title{
Establishment and long-term culture of human cystic fibrosis endothelial cells
}

\author{
Roberto Plebani ${ }^{1,2}$, Romina Tripaldi ${ }^{2,3}$, Paola Lanuti ${ }^{2,3}$, Antonio Recchiuti ${ }^{1,2}$, Sara Patruno ${ }^{1,2}$, Sara Di Silvestre ${ }^{1,2}$, \\ Pasquale Simeone ${ }^{2,3}$, Marco Anile ${ }^{4}$, Federico Venuta ${ }^{4}$, Marco Prioletta ${ }^{1}$, Felice Mucilli ${ }^{1}$, Paola Del Porto ${ }^{5}$, \\ Marco Marchisio ${ }^{2,3}$, Assunta Pandolfi ${ }^{1,2}$ and Mario Romano ${ }^{1,2}$
}

Endothelial cell (EC) dysfunction has been reported in cystic fibrosis (CF) patients. Thus, the availability of CF EC is paramount to uncover mechanisms of endothelial dysfunction in CF. Using collagenase digestion, we isolated cells from small fragments of pulmonary artery dissected from non-CF lobes or explanted CF lungs. These cells were a heterogeneous mixture, containing variable percentages of EC. To obtain virtually pure pulmonary artery endothelial cells (PAEC), we developed an easy, inexpensive, and reliable method, based on the differential adhesion time of pulmonary artery cells collected after collagenase digestion. With this method, we obtained up to 95\% pure non-CF and CF-PAEC. Moreover, we also succeed at immortalizing both PAEC and CF-PAEC, which remained viable and with unchanged phenotype and proliferation rate over the 30th passage. These cells recapitulated cystic fibrosis transmembrane conductance regulator expression and functions of the parental cells. Thus, we isolated for the first time endothelial cells from CF patients, providing a valuable tool to define the emerging role of EC in CF lung and vascular disease.

Laboratory Investigation (2017) 97, 1375-1384; doi:10.1038/labinvest.2017.74; published online 31 July 2017

The vascular endothelium is a metabolically active compartment that regulates a number of key homeostatic processes, including hemostasis and blood coagulation, the vascular tone and the immune-inflammatory response. ${ }^{1,2}$ A dysfunctional endothelium is the hallmark of a variety of pathologic conditions, being atherosclerosis and thrombosis the best known. ${ }^{2}$ However, endothelial dysfunction has also a profound impact on the inflammatory response and its resolution, as it promotes leukocyte recruitment and consequent tissue damage. ${ }^{3}$

Cystic fibrosis (CF) is a genetic disease caused by mutations in the cystic fibrosis transmembrane conductance regulator (CFTR) gene on chromosome $7,{ }^{4}$ being phenyl-alanine 508 deletion (F508del) present in $\sim 70 \%$ of $\mathrm{CF}$ patients. A dysfunctional CFTR alters profoundly $\mathrm{Cl}^{-}$and $\mathrm{HCO}^{-}$ secretion, resulting in the deprivation of the periciliary fluid volume in the airways, with impairment of the mucociliary clearance and aberrant infection and neutrophilic inflammation that lead to respiratory insufficiency and death. ${ }^{5}$
Although the respiratory epithelium is primarily affected by CFTR dysfunction, the mechanisms of the aberrant, uncontrolled lung inflammation in CF are incompletely known. The observation that CFTR is expressed and biologically active in a wide spectrum of cells, including platelets ${ }^{6}$ and leukocytes, ${ }^{7,8}$ has opened new perspective for the definition of the mechanisms underlying CF lung disease.

EC also express CFTR. ${ }^{9}$ We have previously documented that CF patients display peripheral signs of endothelial perturbation, which correlates with loss in respiratory function. ${ }^{10}$ Moreover, CFTR dysfunction is associated with a variety of morphological and functional alterations in endothelial cells (Totani et al, unpublished data). Along these lines, changes in barrier function have been recently reported in endothelial cells with a dysfunctional CFTR. ${ }^{11}$

Main aim of this study was to develop a simple, inexpensive, and reliable methodology to obtain virtually pure and long-term maintained EC from CF patients. This, as first step to develop research aimed at elucidating the

\footnotetext{
'Department of Medical, Oral and Biotechnological Sciences, "G. d'Annunzio" University of Chieti-Pescara, Chieti, Italy; ${ }^{2}$ Center on Aging Sciences and Translational Medicine (CeSI-MeT), "G. d'Annunzio" University of Chieti-Pescara, Chieti, Italy; ${ }^{3}$ Department of Medicine and Aging Sciences, University "G. d'Annunzio" University of Chieti-Pescara, Chieti, Italy; "Department of Thoracic Surgery, Sapienza University, Rome, Italy and ${ }^{5}$ Department of Biology and Biotechnology "Charles Darwin" Sapienza University, Rome, Italy

Correspondence: Dr R Plebani, PhD or Professor M Romano, MD, Center on Aging Sciences and Translational Medicine (CeSI-MeT), "G. d'Annunzio" University of Chieti-Pescara, Cheti, Italy.

E-mail: roberto.plebani@unich.it or mromano@unich.it

Received 28 December 2016; revised 11 May 2017; accepted 18 May 2017
} 
pathobiology of CFTR in EC and the involvement of EC in CF inflammation and vascular pathophysiology.

\section{MATERIALS AND METHODS Isolation and Standard Culture Conditions of Pulmonary Artery Cells}

Pulmonary arteries (PA) were excised from explanted CF lungs or from non-CF lungs during lobectomy. All donors signed an informed consent form. After careful removal of fat and connective tissue, segmental branches of the PA were cut into 2-cm pieces, whereas subsegmental branches were cut into 3-4 cm pieces. Fragments were stored in phosphatebuffered saline (PBS) containing linezolid $(250 \mu \mathrm{g} / \mathrm{ml})+$ colistin $(50 \mathrm{U} / \mathrm{ml})+$ cotrimoxazole $(25 \mu \mathrm{g} / \mathrm{ml})+$ amphotericin $B$ $(25 \mu \mathrm{g} / \mathrm{ml})$ (LCCA). Within $20 \mathrm{~h}$ from surgery, arteries were rinsed three times with $0.9 \%$ saline containing penicillin/ streptomycin $(\mathrm{P} / \mathrm{S})(1 \%)$ and amphotericin B $(25 \mu \mathrm{g} / \mathrm{ml})$ to remove blood cells. Arteries were then incubated $(20 \mathrm{~min}$, $37^{\circ} \mathrm{C}, 5 \% \mathrm{CO}_{2}$ ) with $2 \mathrm{mg} / \mathrm{ml}$ type II collagenase (Worthington Biochem, Lakewood, NJ, USA) diluted with calcium and magnesium-free PBS. Immediately after the incubation, arteries were moved into a Petri dish $(100 \mathrm{~mm})$ containing endothelial cell culture medium (50\% vol/vol DMEM-M199 medium, supplemented with 10\% FBS, $1 \%$ L-glutamine, $1 \%$ penicillin/streptomycin, 1\% ECGF, 1\% heparin), massaged with a spatula followed by gentle shaking. Cells were collected by centrifugation ( $330 \mathrm{~g}$ for $7 \mathrm{~min}$ at room temperature), seeded on fibronectin $\left(1 \mathrm{mg} / \mathrm{cm}^{2}\right)$-coated plates ${ }^{12}$ and maintained in the aforementioned culture medium supplemented with LCCA, whose concentration was halved every 2 days until zero, when only the P/S standard concentration was maintained.

\section{Culture Condition during Selection}

During selection, cells were maintained with $50 \% \mathrm{vol} / \mathrm{vol}$ DMEM-medium 199 supplemented with 10\% fetal bovine serum (FBS), $100 \mathrm{U} / \mathrm{ml}$ penicillin, $100 \mathrm{micro} / \mathrm{ml}$ streptomycin, and $1 \%$ glutamine. After the explant, cells $\left(1 \times 10^{6}\right)$ were seeded in two $100 \mathrm{~mm}$ gelatin-coated dishes. At confluence, cells from both dishes were detached with trypsin and seeded in a new plate. In this phase, $10 \mathrm{~min}$ after seeding, the majority of cells remained in the supernatant, while a small number of cells $(\sim 8-10 \%)$ was already adherent. At this time point, the supernatant was removed from one of the plates and replaced with fresh medium, whereas the second plate was left untouched. This adherence time-based selection procedure was repeated at each passage until a virtually pure population of pulmonary artery endothelial cells was obtained. For each selection step, images were taken using an optical microscope.

\section{Immunophenotyping}

Cells $\left(5 \times 10^{5} /\right.$ sample $)$ were immunophenotyped by flow cytometry as previously reported. ${ }^{13}$ Briefly, samples were centrifuged ( $400 \mathrm{~g}, 10 \mathrm{~min}$ at room temperature) and washed with $2 \mathrm{ml}$ of stain buffer with bovine serum albumin (Becton Dickinson Biosciences - BD Biosciences, San Jose, CA, USA). For EC identification, surface staining was carried out by adding to the pellet $10 \mu \mathrm{l}$ fluorescein isothiocyanate (FITC)conjugated CD31 (BD Biosciences, clone WM59) and $10 \mu \mathrm{l}$ R-phycoerythrin (PE)-conjugated CD146 (BD Biosciences, clone $\mathrm{P} 1 \mathrm{H} 12)$. Isotype-matched antibodies were used as negative controls. Samples were incubated for $30 \mathrm{~min}$ at $4{ }^{\circ} \mathrm{C}$, washed with $2 \mathrm{ml}$ stain buffer with BSA (BD Biosciences) and suspended with $0.5 \mathrm{ml}$ FACSFlow (BD Biosciences). Flow cytometric analysis was carried out by recording $2 \times 10^{4}$ events/sample on a FACSCanto II cytometer (BD Biosciences). The following antibodies were used to determine the phenotype of non-EC contained in the mixed population collected from pulmonary artery: CD14 FITC from Miltenyi Biotec, Calderara di Reno (BO), Italy $(3 \mu \mathrm{l})$; CD44 FITC from BD $(3 \mu \mathrm{l})$; CD13 FITC from Ancell Corporation, Bayport, MN, USA $(1 \mu \mathrm{l})$; CD105 FITC from Ancell $(1 \mu \mathrm{l})$; CD166 FITC from Ancell $(1 \mu \mathrm{l})$; HLAABC Alexa 488 from BD $(10 \mu \mathrm{l})$; CD29 PE from Ancell $(1 \mu \mathrm{l})$; EPCAM PE Cy5.5 from BD Biosciences $(10 \mu \mathrm{l})$; and CD73 PE from BD Bioscience $(5 \mu \mathrm{l})$. Antibodies were titrated under assay conditions, in order to obtain optimal dilutions. Instrument performances and data reproducibility were maintained and checked by using the Cytometer Setup and Tracking Module (BD Biosciences). Gates were assessed on the basis of unstained and isotype controls. Compensation was calculated by running CompBeads (BD Biosciences). Data were analyzed using the FACSDiva v 6.1.3 (BD Biosciences) software.

\section{Confocal Microscopy}

Cells $\left(2 \times 10^{4}\right)$ were seeded on glass coverslips pre-coated with $1.5 \%$ gelatin and fixed, after $24 \mathrm{~h}$, with $4 \%$ paraformaldehyde. Cells were permeabilized with $0.5 \%$ saponin and stained overnight at $4{ }^{\circ} \mathrm{C}$ with primary anti VWF (MA5-14029, Thermo Fisher Scientific, Waltham MA, USA, 1:100 dilution) and anti CD31 (M0823, Dako, 1:25 dilution) antibodies, or $1 \mathrm{~h}$ at room temperature with an anti-CFTR antibody (MAB25031, clone 24-1, R\&D Systems, Minneapolis, MN, USA, 1:25 dilution), followed by secondary goat anti-mouse IgG conjugated with Alexa Fluor $488\left(1 \mathrm{~h}\right.$ at $\left.4{ }^{\circ} \mathrm{C}\right)$. Cells were incubated with DRAQ-5 together with the secondary antibody for nuclear staining. High definition images were acquired by a LSM-510 META (Carl Zeiss, Jena, Germany) confocal microscope.

\section{Matrigel Assay}

A tube formation assay was performed as functional readout of the endothelial phenotype. ${ }^{14}$ To this end, matrigel (BD Biosciences) was aliquoted on a 12-well tissue culture plate and allowed to solidify for $1 \mathrm{~h}$ at $37^{\circ} \mathrm{C}$. Cells $\left(10^{5}\right)$ were seeded in $200 \mu \mathrm{l}$ culture medium w/o serum. Wells were observed under a contrast phase microscope and images taken at 3,6 , and $8 \mathrm{~h}$ after seeding. Replicates were performed for each assay condition. 


\section{Immortalization of PAEC and CF-PAEC}

MuLV retroviral particles were obtained by co-transfection of HEK-293T with plasmids coding for MuLV gag and pol (pUMVC, gift from Bob Weinberg, Addgene plasmid \#8449), envelope proteins (pCMV-VSV-G, gift from Bob Weinberg, Addgene plasmid \#8454), ${ }^{15}$ and pBABE-puro-SV40 LT (gift from Thomas Roberts, Addgene plasmid \#13970) ${ }^{16}$ modified Scott Dessain and Hua-yin Yu, Weinberg Lab protocol (https://www.addgene.org/static/data/84/61/162606cc-af64-11e090fe-003048dd6500.pdf). Cells were infected with retroviral particles in the presence of $5 \mu \mathrm{g} / \mathrm{ml}$ Polybrene (Sigma-Aldrich) and selected with Puromycin $(1.5 \mu \mathrm{g} / \mathrm{ml})$ (InvivoGen). Puromycin-resistant non-CF and CF-PAEC were stained with a FITC-conjugated anti-CD31 antibody (BD Biosciences, clone WM59), as reported above, under sterile conditions. $\mathrm{CD}^{+} 1^{+}$cells were sorted using a FACSAria III cell sorter, BD, $100 \mu \mathrm{m}$ nozzle.

\section{CFTR Activity}

A total of $2 \times 10^{4}$ immortalized PAEC and CF-PAEC were seeded in a 24-well plate. After $24 \mathrm{~h}$, cells were washed three times with a $5 \%$ glucose solution and placed in a Synergy H1 Multi-Mode Reader instrument (BioTek, Winooski, VT, USA) for background fluorescence reading (excitation at $530 \mathrm{~nm}$, emission at $630 \mathrm{~nm}$ wavelength). Then, $250 \mathrm{nM}$ of the voltage-sensitive probe Bis-(1-3-diethylthyobarbituric acid) trimethine oxonol $\left(\operatorname{DISBAC}_{2}(3)\right)$ was added to the cells and the fluorescence value was recorded every $15 \mathrm{~s}$ for $5 \mathrm{~min}$ to set the baseline. After $5 \mathrm{~min}$, the cells were either left unstimulated or exposed to $200 \mu \mathrm{M}$ 8-bromo cAMP and $10 \mu \mathrm{M}$ forskolin to activate the CFTR channel and fluorescence values were recorded every $15 \mathrm{~s}$ for additional $50 \mathrm{~min}$. Fluorescence values from unstimulated cells were subtracted from values obtained with cAMP and forskolinstimulated cells.
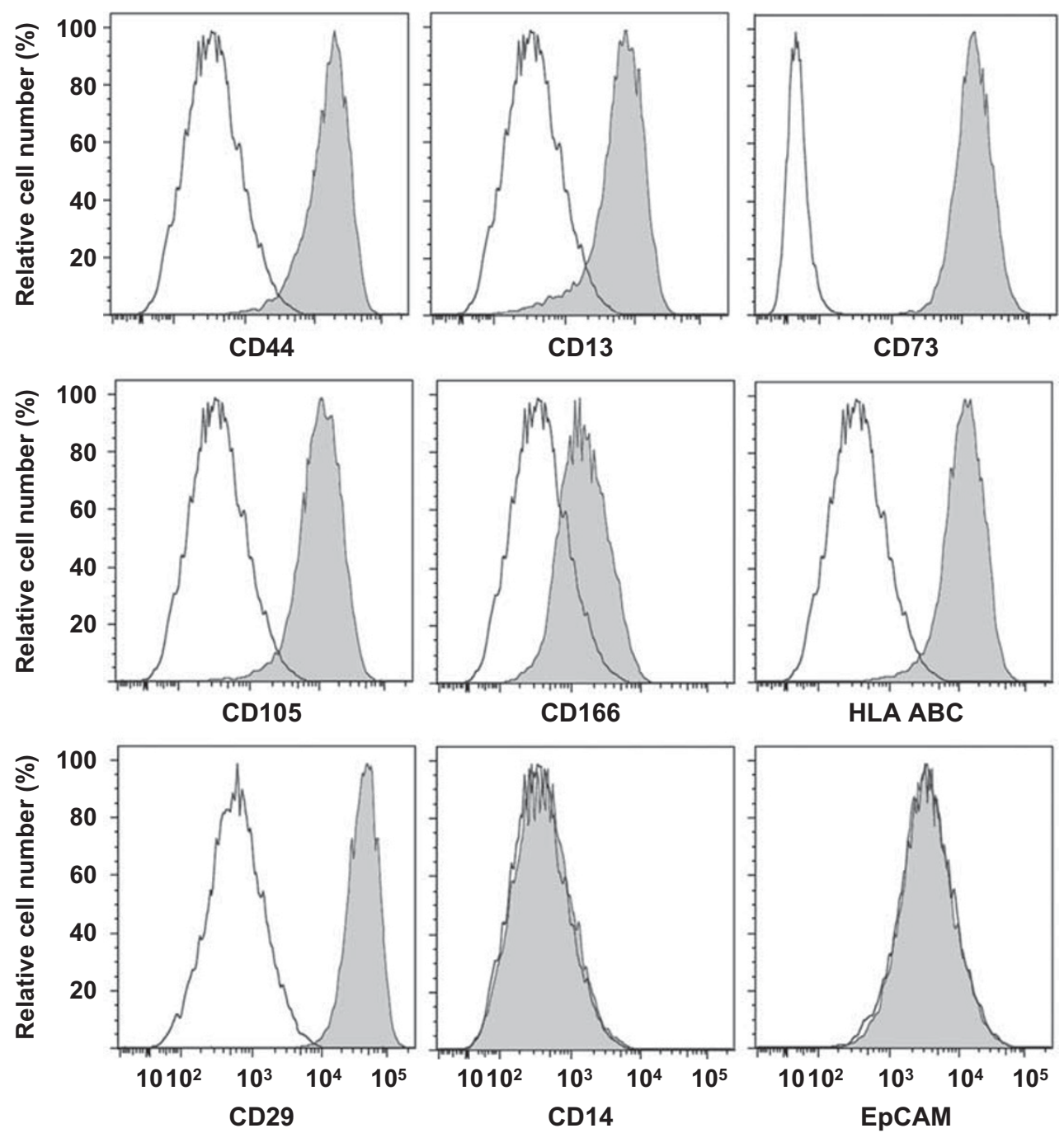

Figure 1 Characterization of non-endothelial pulmonary artery cells. Cell surface staining of non-endothelial cell populations for CD14 FITC, CD44 FITC, CD13 FITC, CD105 FITC, CD166 FITC, HLA ABC Alexa 488, CD29 PE, EpCAM PE Cy5.5, and CD73 PE. Red histograms represent stained samples while blue histograms are the related isotypic controls. Results are representative of three separate experiments. 
a
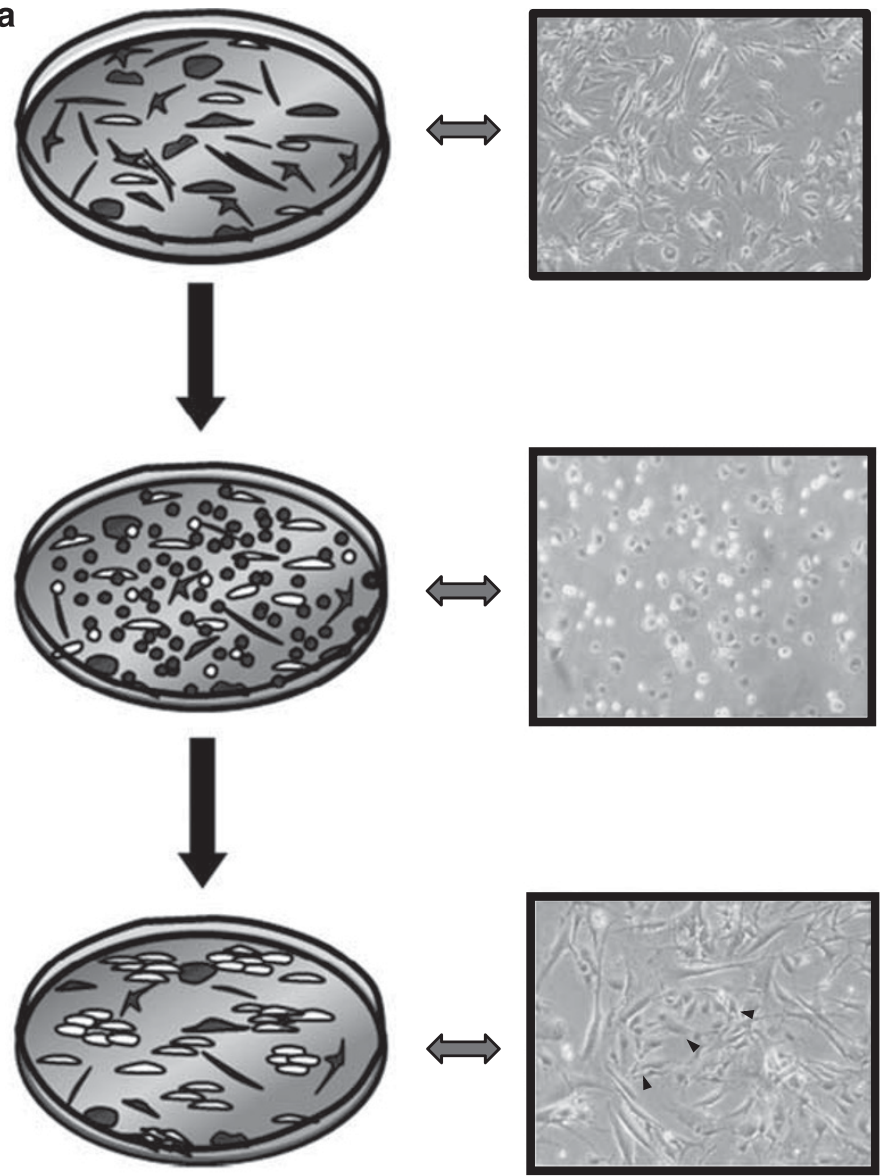
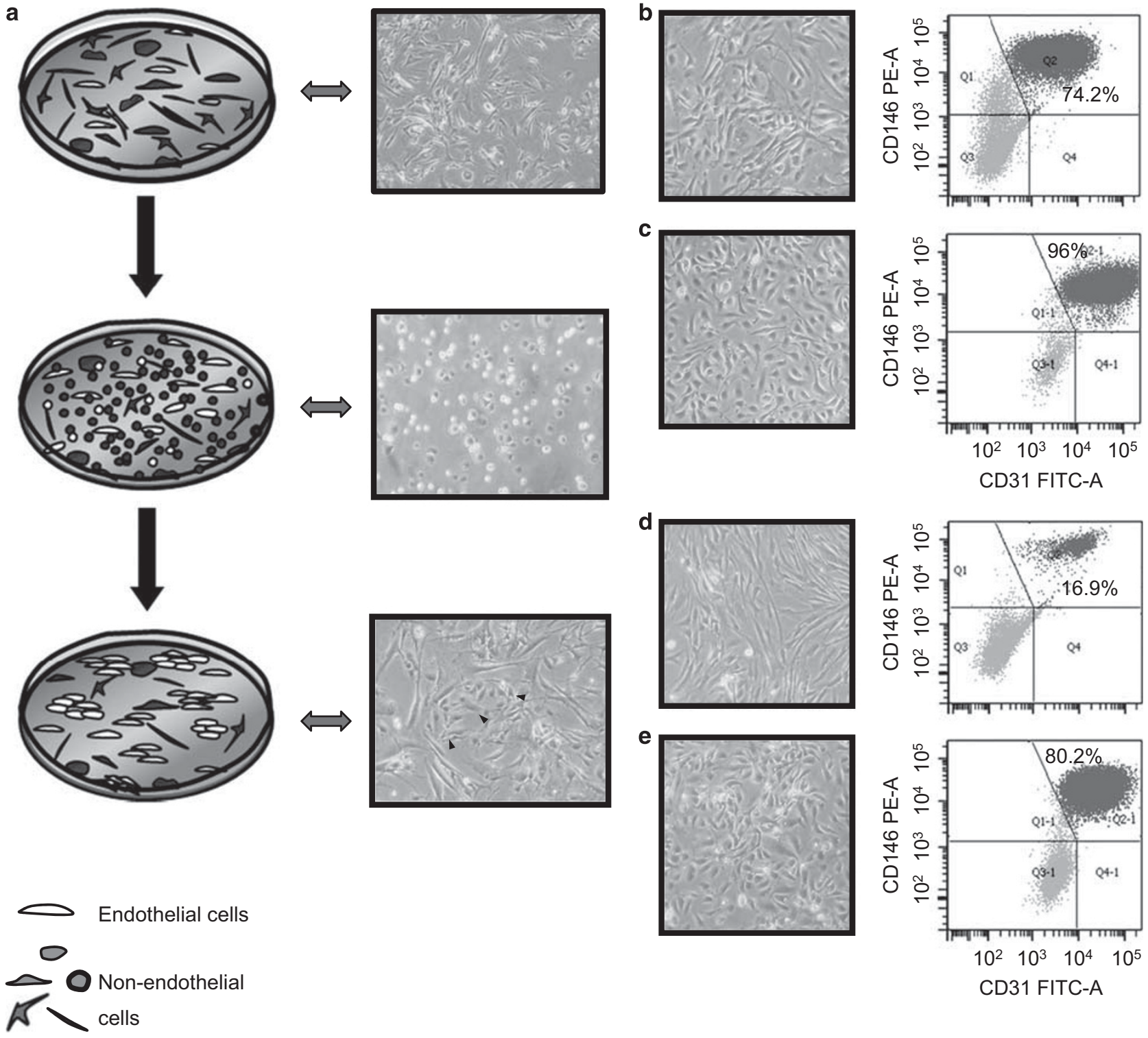

Figure 2 Selection strategy. (a) Cell population after explants from non-CF lungs (top panel). Pulmonary artery cells 10 min after being seeded into gelatin-coated dishes (middle panel). Cell morphology after one selection cycle (bottom panel). Arrows indicate the cells with endothelial-like morphology. (b-e) Flow cytometry quantitation of cells carrying the endothelial phenotype within preparations of cells isolated from two different donors. Cell morphology, evaluated by light microscopy, is shown in the left panels, whereas CD146/CD31 staining, assessed by flow cytometry, is illustrated in the dot plots of the right panels. Cells from donor 1 after collection (b) and two steps of selection (c). Cells from donor 2 after collection (d) and three selection cycles (e). The percentage of $\mathrm{CD}_{146} / \mathrm{CD} 31^{+}$is reported in the upper right corner of the right panels.

\section{Transendothelial Electric Resistance (TEER)}

Immortalized PAEC and CF-PAEC were seeded in gelatincoated $0.4 \mu \mathrm{m}$ polyester membrane transwell inserts (Corning, Tewksbury MA, USA) at $4 \times 10^{4}$ cells/well. At cell confluence, TEER as evaluated using an EVOM analyzer. Measurements were taken in three different sections of the insert. Inserts without cells or containing untreated cells were used as blank and baseline resistance, respectively. TEER values were obtained by subtracting the blank from the resistance recorded in the insertcontaining cells.

\section{Statistical Analysis}

Statistical significance was assessed using one-way ANOVA with Bonferroni's post hoc test. $P$-values $<0.05$ were considered as statistically significant.

\section{RESULTS}

\section{PAEC Enrichment during the Selection Procedure}

Pulmonary artery explants contained a mixed cell population with different shapes, mainly fibroblast-like and, at a lower extent, reminiscent of endothelial morphology. ${ }^{17}$ These cells were immunophenotyped using several markers (Figure 1). 


\section{a}

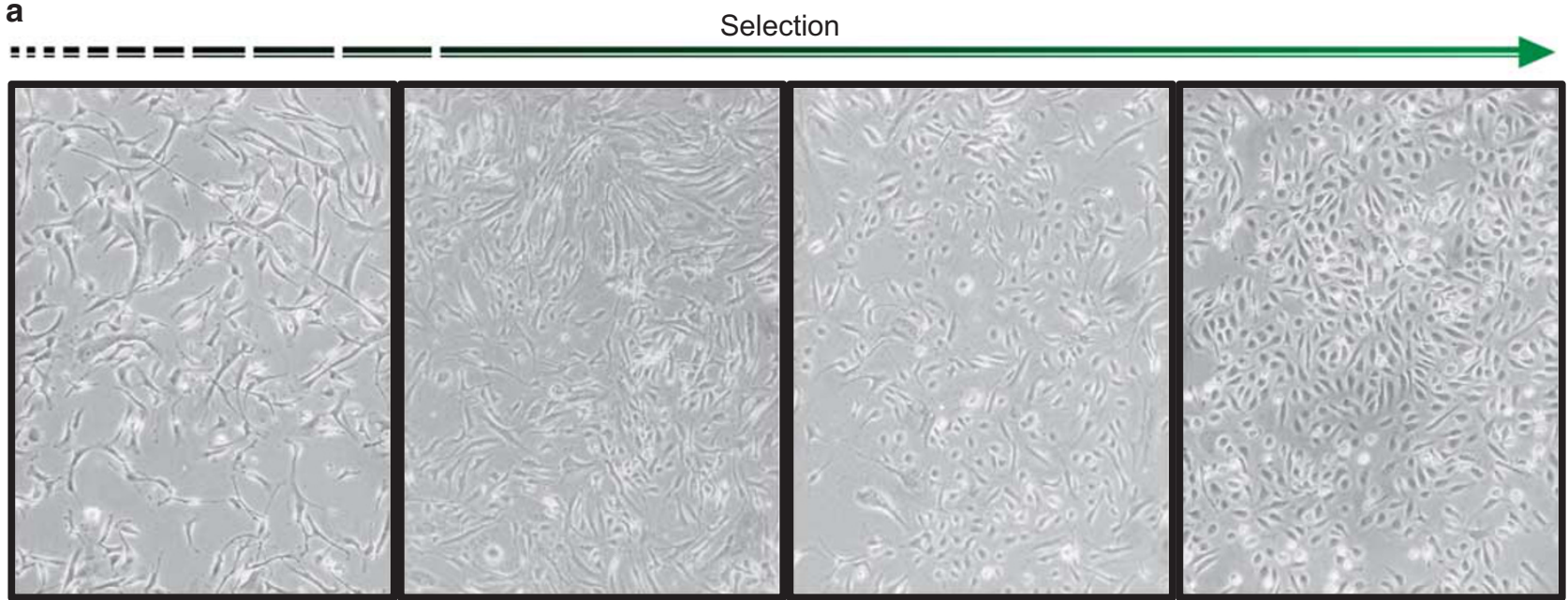

b

Before selection

After 3 selection steps
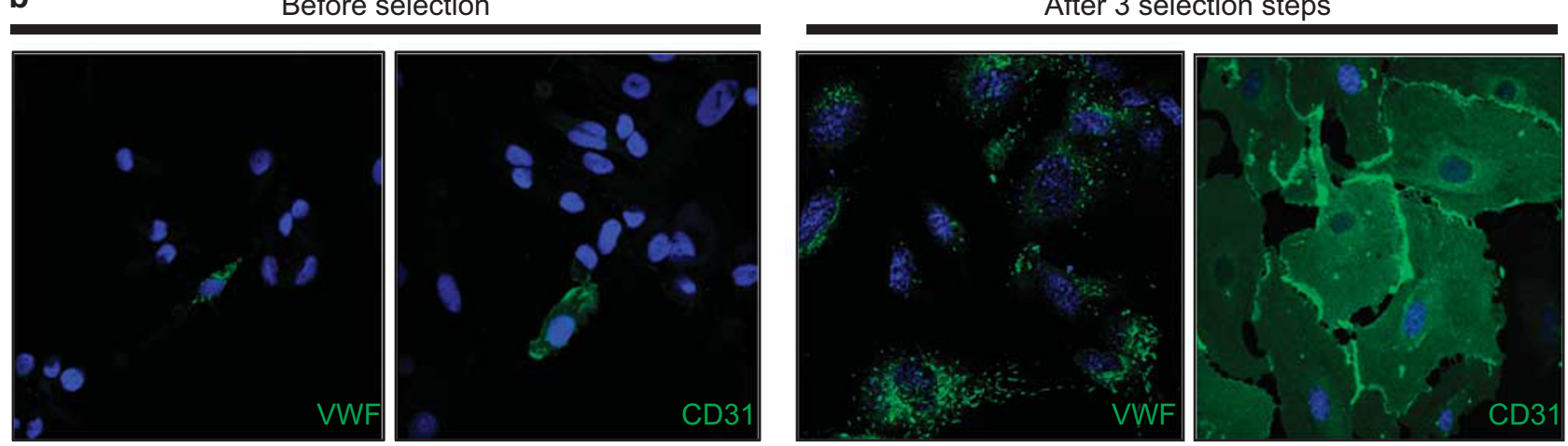

Figure 3 Characterization of CF-PAC. (a) CF cell morphology at 0, 1, 2, and 3 selection passages (from left to right). (b) VWF and CD31 confocal staining of CF cells at step 0 (left panel) and after three selection passages (right panel).

Cells isolated from three different donors consistently stained positive for CD44, CD13, CD73, CD105, CD166, HLA-ABC, and $\mathrm{CD} 29$, whereas they resulted negative for the monocyte/ macrophage marker CD14, as well as for epithelial EpCAM (Figure 1).

These populations clearly showed a different adhesion time to gelatin-coated dishes, being cells with the endothelial-like morphology faster to adhere (Figure 2). Fast-adhering cells stained positive for both CD31 and CD146, two known endothelial antigens analyzed by flow cytometry ${ }^{18,19}$ (Figures $2 b-e)$. The percentage of these cells within the explants from two non-CF donors was variable, ranging from 74.2 (Figure 2b) to $16.9 \%$ (Figure 2d). These percentages increased to 96 (Figure 2c) and $80.2 \%$ (Figure 2e), respectively, after two and three selection steps. These results indicate that PAEC can be separated from mixed cell cultures based on their adhesion time.

\section{CF-PAEC Isolation and Purification}

Since CF-PAEC are of wide interest to understand mechanisms of inflammation and vascular complications in $\mathrm{CF},{ }^{20,21}$ we applied the selection procedure to cells isolated from a lung explanted from a $\mathrm{CF}$ patient carrying the genotype G542X/1717-1G- > A genotype. Changes in morphology of the overall cell population during selection is illustrated in Figure 3a, whereas von Willebrand Factor (vWF) and CD31 staining, assessed by confocal microscopy, is shown in Figure 3b. It is evident that during selection, the percentage of cells characterized by typical endothelial morphology (arrows) increased progressively (Figure 3a). Consistent with this, only few cells within the initial pool (before selection) stained positive for vWF or CD31 (Figure 3b, left panel), while after the third selection step, almost the whole cell population expressed both antigens (Figure $3 \mathrm{~b}$, right panel). We obtained comparable results ( 94\% $\left.\mathrm{CD} 146^{+} / \mathrm{CD} 31^{+}\right)$ with surgical specimens collected from another $\mathrm{CF}$ patient carrying the F508del/F508del genotype (results not shown).

To further characterize the enrichment of the endothelial compartment during the selection process, CF cells before (step 0) and after three selection passages (step 1, step 2 and step 3, respectively) were analyzed by flow cytometry. As shown in Figure 4a, at step 0 cells displayed heterogeneous antigen expression as some were $\mathrm{CD} 146^{-} / \mathrm{CD}^{-} 1^{-}$, some were $\mathrm{CD} 146^{+} / \mathrm{CD} 1^{-}$, and only $17.7 \%$ of total cells expressed the 

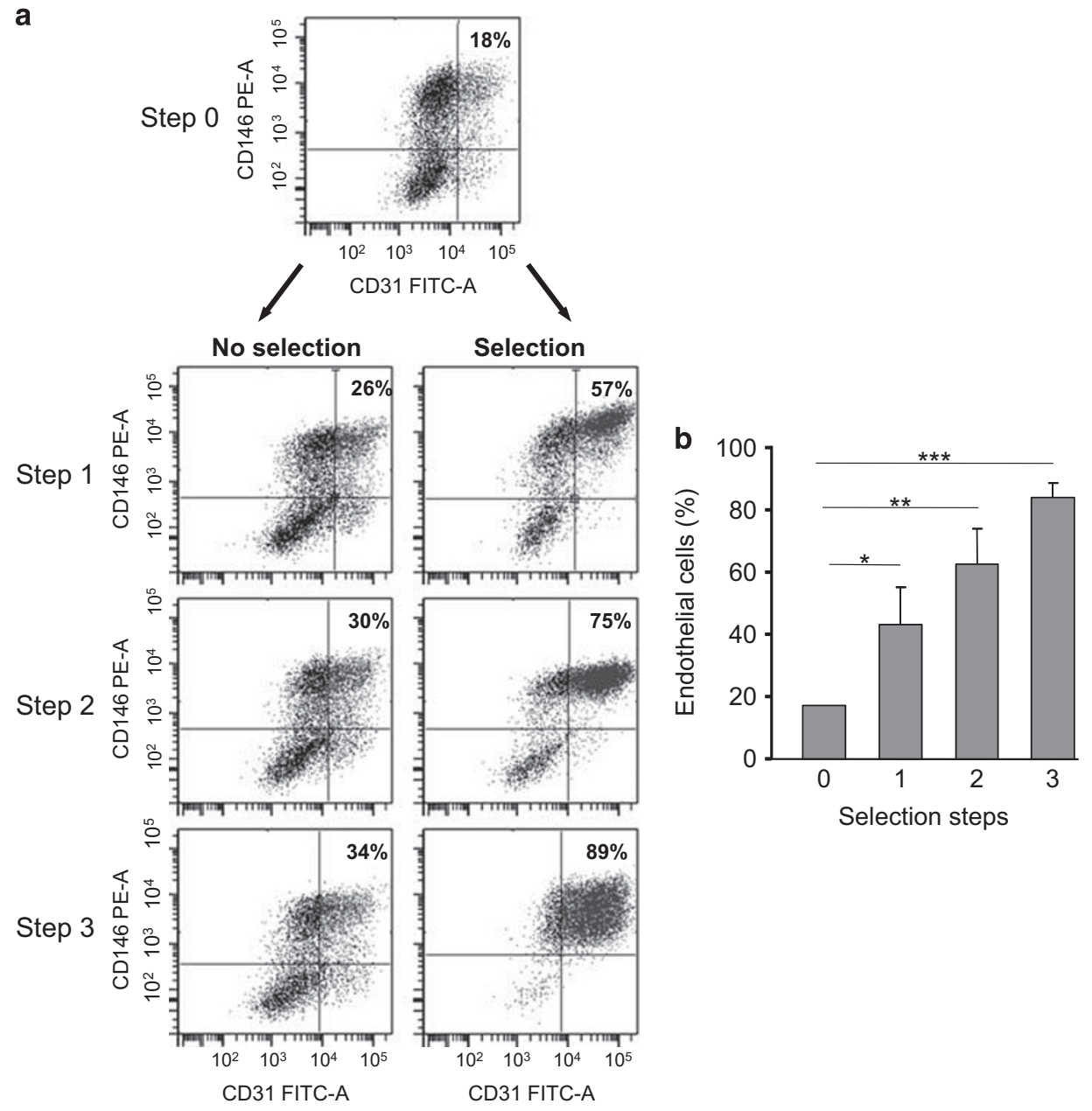

Figure 4 CF-PAEC enrichment during the selection procedure. (a) Flow cytometry analysis of CD $146^{+} / \mathrm{CD} 31^{+}$cells in the initial pool (step 0), and after 1 , 2 , and 3 selection steps. Plots are representative of triplicates. (b) Bars represent mean \pm s.e.m. of $\mathrm{CD} 146^{+} / \mathrm{CD} 31^{+}$cells from $n=3$ plates $\left({ }^{*} P=0.05\right.$; ${ }^{* *} P=0.01 ;{ }^{* * *} P=0.001$ versus the initial pool).

EC phenotype $\left(\mathrm{CD} 146^{+} / \mathrm{CD} 31^{+}\right)$. Cells subjected to selection (right dot plots) displayed a significant increase in the $\mathrm{CD} 146^{+} / \mathrm{CD} 31^{+}$population from 18 to $89 \%$. Data from triplicates are reported in Figure $4 \mathrm{~b}$. On the contrary, the percentage of $\mathrm{CD} 146^{+} / \mathrm{CD} 31^{+}$cells did not change significantly in non-selected cells (Figure $4 \mathrm{a}$, left plots). Notably, the $\mathrm{CD} 146^{+} / \mathrm{CD} 31^{-}$cell population decreased during the selection procedure in both selected and non-selected samples, while $\mathrm{CD} 146^{-} / \mathrm{CD} 31^{-}$cells dramatically decreased from 34.8 to $3.8 \%$ after selection (Figure $4 \mathrm{a}$, right plots).

\section{Tube Formation on Matrigel}

The ability of selected and non-selected cells to form tubular structures on matrigel was also assessed. Notably, as early as $3 \mathrm{~h}$ after seeding, selected CF cells (Figure 5a) showed more evident ability to form tubular structures on matrigel as compared to not-selected cells (Figure 5b). These differences, which were also evident 6 and $8 \mathrm{~h}$ after seeding, clearly indicate that the selected population was enriched in PAEC.
Together with data in Figures 3 and 4, these results indicate that by using a simple procedure is possible to obtain virtually pure CF-PAEC populations.

\section{PAEC and CF-PAEC Immortalization}

Because EC in culture have a very limited life span, due to early replicative senescence, we undertook the task to immortalize PAEC and CF-PAEC carrying the F508del/ F508del genotype. To this end, we infected cells with SV40 large $\mathrm{T}$ antigen and selected puromycin-resistant cells. SV40 gene expression was evaluated after a high number of passages (18 for CF-PAEC and 14 for PAEC) by PCR using the following primers: forward, 5 -GACTCAGGGCATGAAACA GG-3'; reverse, 5'-ACTGAGGGGCCTGAAATGA-3'. The $61 \mathrm{bp}$ PCR product was visualized by agarose $(3 \%)$ gel electrophoresis (Figure 6a). Cells were then sorted using an anti-CD31 antibody to obtain pure EC (Figure 6b). The population purity was rechecked after sorting using flow cytometry. As reported in Figure 6c, both immortalized PAEC 
a

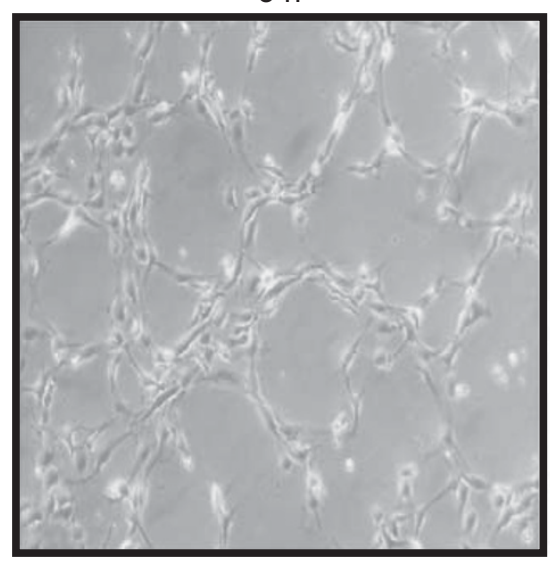

b

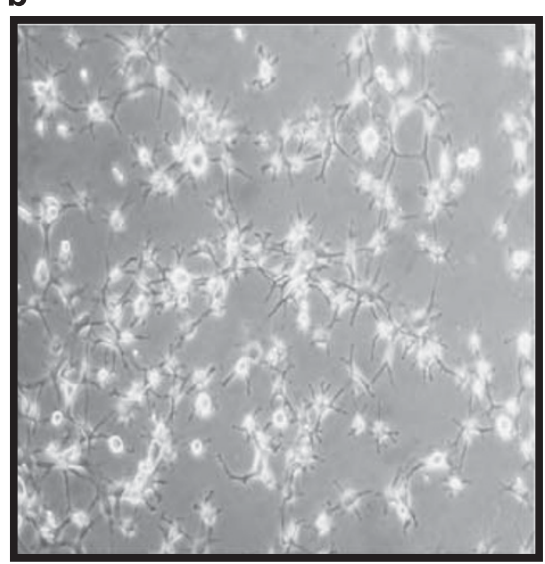

$6 \mathrm{~h}$
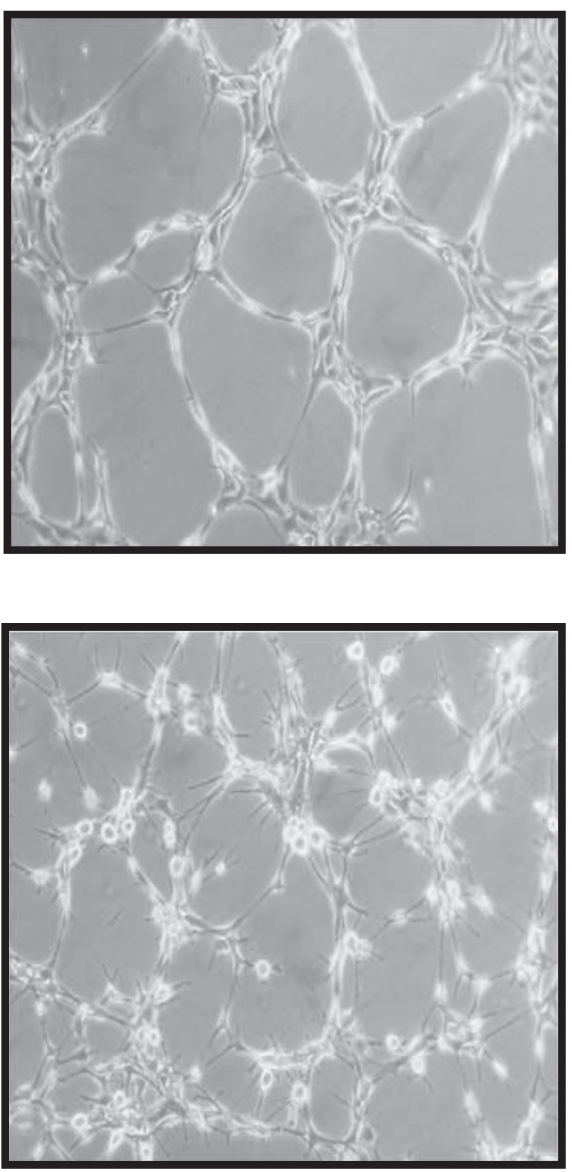

$8 \mathrm{~h}$
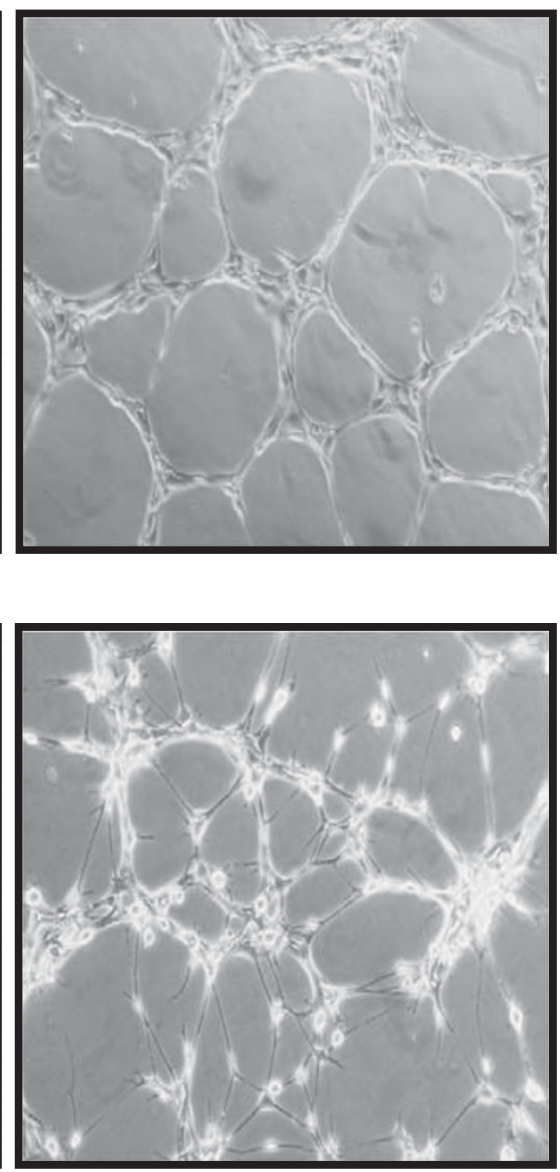

Figure 5 Tube formation assay. Phase-contrast microscopy photographs of CF-PAEC cells cultured on matrigel at varying time after seeding. (a) Selected cells after three passages. (b) Non-selected cells after three passages.

and CF-PAEC maintained the endothelial phenotype $\left(97-99 \% \mathrm{CD} 1^{+} / \mathrm{CD} 146^{+}\right.$cells). These cells were viable and displayed unchanged proliferation rate over the 30th passage.

\section{Evaluation of CFTR Expression and Function in Immortalized PAEC}

We also evaluated CFTR expression and activity in the immortalized cells. Consistent with results with parental cells (Totani et al, unpublished data), immortalized CF-PAEC exhibited reduced membrane CFTR expression compared to PAEC (Figure 7a). This was associated with lower CFTR activity compared to non-CF cells (Figure $7 \mathrm{~b}$ ). A similar reduction was observed in F508del/F508del human CF airway cells (results not shown), confirming that immortalized CF-PAEC are representative of the F508del/F508del phenotype. Moreover, immortalized CF-PAEC displayed reduced TEER compared to PAEC (Figure 7c).

\section{DISCUSSION}

Primary cells isolated from patients are particularly useful to study pathogenetic mechanisms and as tools for personalized medicine. The need for primary cells from individual patients is stringent in genetic disorders such as CF, where $~ 2000$ mutations of the CFTR gene have been reported (http://www. genet.sickkids.on.ca/app).

Recent results, also from our group, are challenging the epithelium-centered vision of CF pathogenesis, as CFTR is expressed by a variety of cell types, where it controls a number of relevant functions. Among these, vascular endothelial cells, which are dysfunctional in CF. ${ }^{10,11,22}$

Despite their potential pathogenetic role in CF, CF-PAEC had never been isolated before. Here we report a simple, inexpensive, and reliable procedure to obtain a virtually pure population of EC from pulmonary arteries of CF patients. To achieve this goal, we had to overcome two main technical problems: the high bacterial contamination of the surgical specimens, due to chronic CF lung infection ${ }^{23}$ and the presence of abundant non-endothelial cells among those collected after collagenase digestion of the PA fragments (Figure 2). A variable number of these cells expressed the $\mathrm{CD} 146^{+} / \mathrm{CD} 31^{-}$phenotype (Figures 2 and 4 ). They also expressed CD44, CD13, CD73, CD105, CD166, and CD29 
a

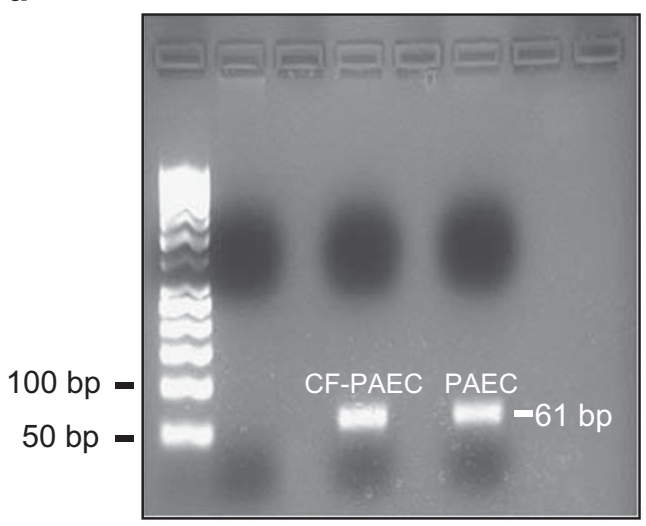

c CF-PAEC b

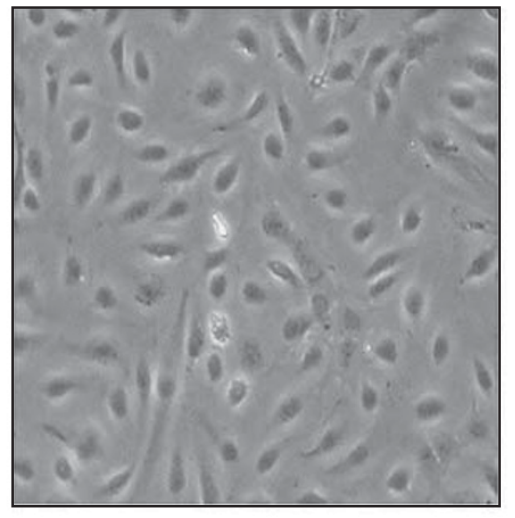

CF-PAEC

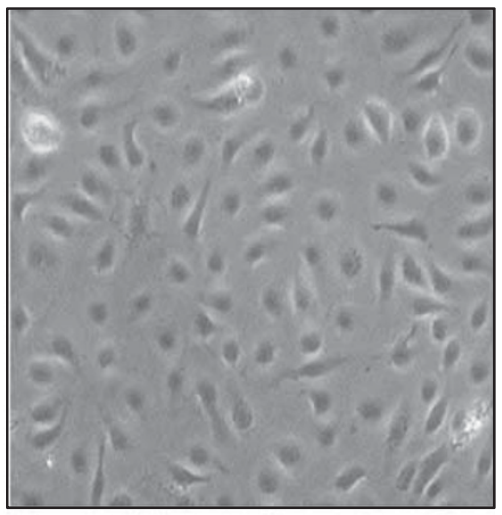

PAEC
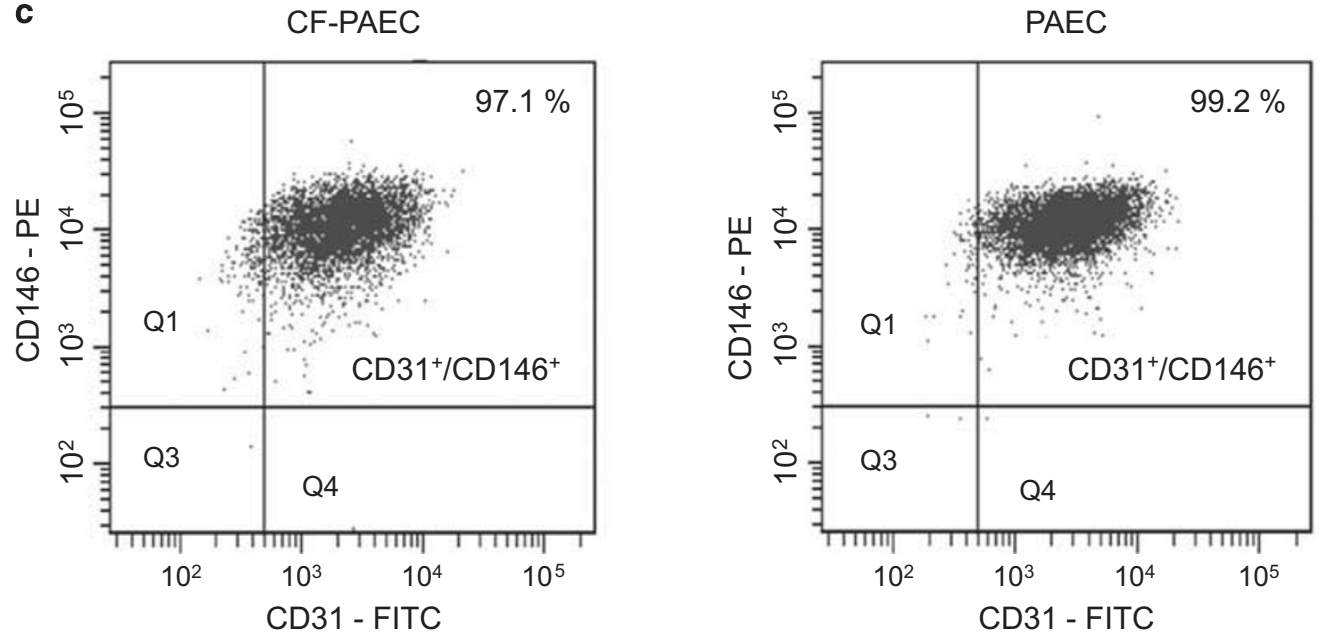

Figure 6 Immortalization of PAEC and F508del/F508del CF-PAEC. Cells and were infected with SV40LT and selected for puromycin resistance. SV40 gene expression was evaluated after a high number of passages (18 for CF-PAEC and 14 for PAEC) by PCR. The PCR product (61 bp) was visualized by electrophoresis on a 3\% agarose gel (a). Both CF-PAEC and PAEC were then sorted using an anti-CD31 antibody and analyzed, at passage 22 for CFPAEC and 24 for PAEC, by light microscopy (b) and flow cytometry (CD31-FITC/CD 146-PE staining) (c).

(Figure 1), which is consistent with a mesenchymal stem cell phenotype. ${ }^{24}$ It has been reported that, when cultured under endothelial conditions, mesenchymal stem cells could differentiate into endothelial cells. ${ }^{25,26}$ Although this may explain the spontaneous enrichment in $\mathrm{CD} 31^{+} / \mathrm{CD} 146^{+}$even in populations not subjected to the selection procedure (Figure 4), further studies are required to fully elucidate this point.

Our method, based on the shorter adhesion time of EC compared to other cell types present in the mixture collected after collagenase digestion of PA (Figures 2, 3 and 4), is rather simple, but it has some caveats, which may influence its outcome. For instance, seeding density after each selection step is relevant, as low density $(\sim 10 \%)$ (Figure 2 ) is more favorable to the identification of EC islands and reduces the number of passages to obtain a good number of pure EC. This is a key issue in experiments with primary cells, particularly EC, which maintain the original phenotype for a limited number of subculturing cycles (www.atcc.org). On the other side, the adhesion time of EC under optimal culturing conditions should not exceed $10 \mathrm{~min}$. Light microscopy monitoring during the procedure is always advised.

We applied our method, first tested with numerous nonCF samples, to two isolates of CF-PAEC from patients with different genotype. In both cases, we observed a great enrichment in EC, obtaining a virtually pure EC population within three passages. In addition of carrying a typical endothelial phenotype (Figures 3 and 4), these cells generated tubular structures when grown in a matrigel matrix (Figure 5), this confirming their endothelial nature. Compared to cell sorting, which is the preferable strategy to obtain pure cell populations, the present method has the advantage of higher yields as $\sim 50 \%$ of cells from one of the $\mathrm{CF}$ isolates was lost during sorting (results not shown), and reduced costs and risk of contamination. These features are particularly relevant in the case of CF-PAEC, which are difficult to obtain 
a

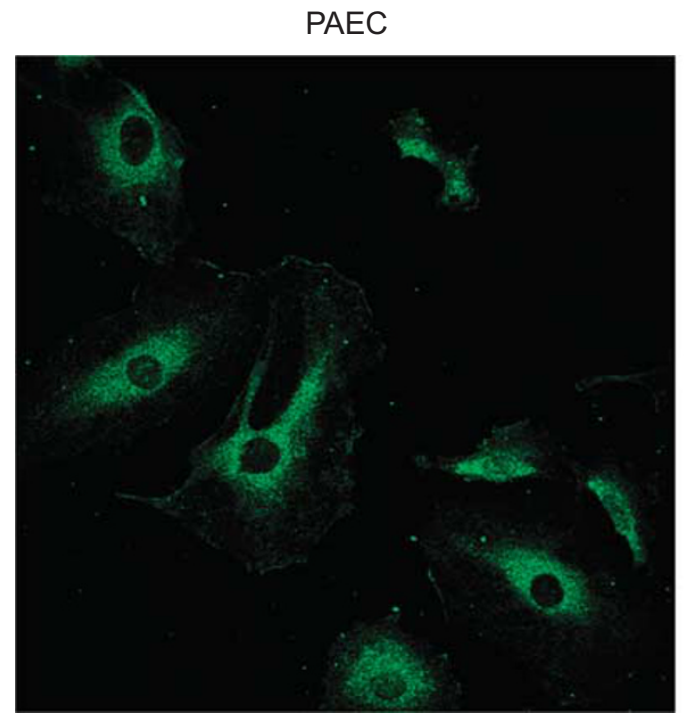

b

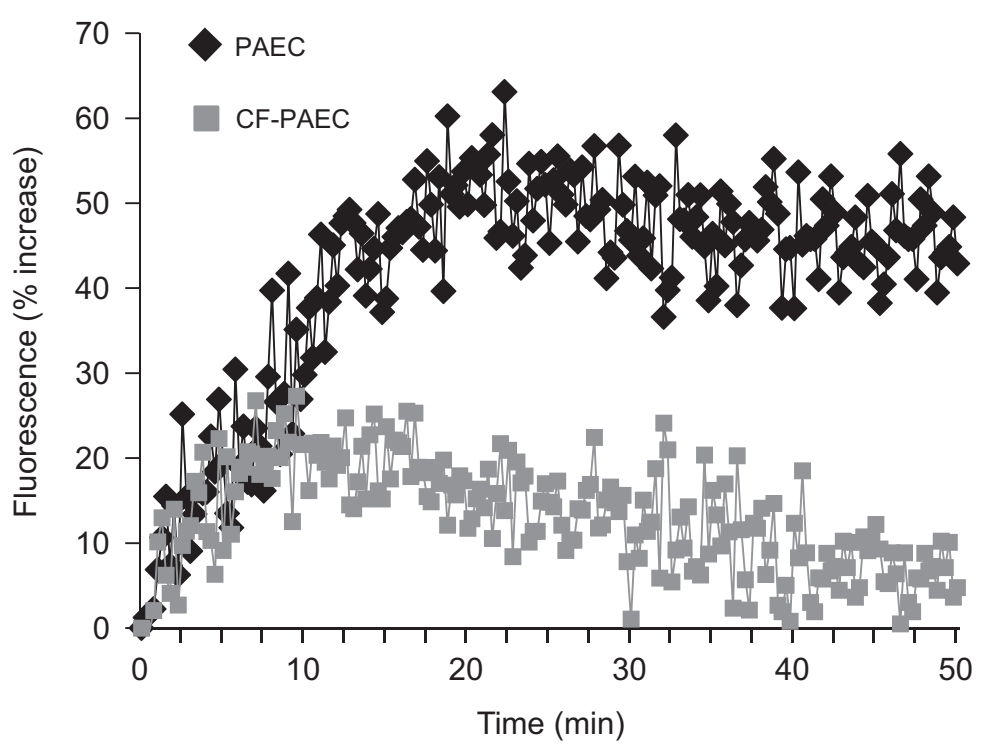

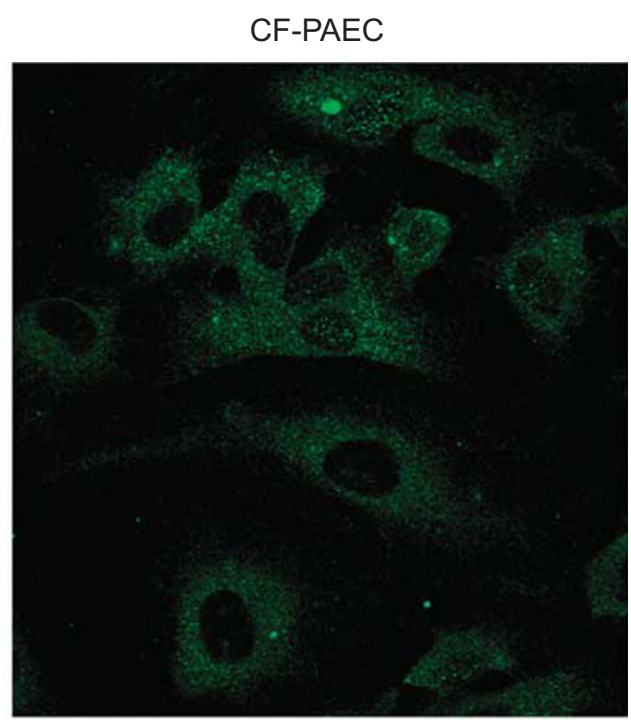

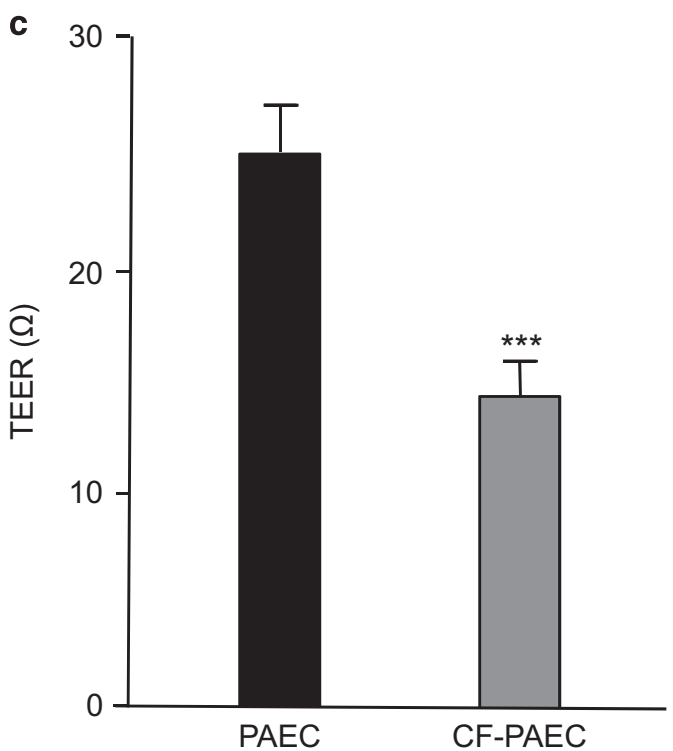

Figure 7 CFTR expression and activity in immortalized PAEC and CF-PAEC. (a) Immortalized PAEC (left panel) and CF-PAEC (right panel) were seeded on glass coverslips and incubated for $1 \mathrm{~h}$ at room temperature) with an anti-CFTR antibody (MAB25031, clone 24-1, R\&D, 1:25 dilution), followed by secondary goat anti-mouse IgG conjugated with Alexa Fluor 488 ( $1 \mathrm{~h}$ at room temperature). High definition images were acquired by a LSM-510 META (Carl Zeiss, Jena, Germany) confocal microscope. (b) CFTR activity was evaluated using the DiSBAC 2 (3) fluorescent probe (see 'Materials and Methods' section for details). Data points illustrate fluorescence readings starting from cell stimulation with 8 -bromo cAMP (200 $\mu \mathrm{M})$ and forskolin (10 $\mu \mathrm{M})$ subtracted of values from unstimulated cells. (c) TEER was determined using an EVOM analyzer as reported in methods. Data are from two independent experiments with six measurements for each point. ${ }^{* *} P=0.0006$.

from transplant centers and are at high risk of bacterial colonization. Along these lines, the preparation of immortalized CF-PAEC (Figure 6) may help to overcome the limitation of primary EC cultures, providing a valuable tool to investigators from different fields. Indeed, these cells recapitulated the phenotype of the parental cells, being CFTR membrane expression and activity reduced in CF cells (Figure 7). This is consistent with patch clamping measurements in primary PAEC and CF-PAEC reported in a recently submitted manuscript (Totani et al, unpublished results). In that work, we also extensively characterized the mechanisms of endothelial dysfunction in CF. Here we report that similarly to parental cells, the immortalized CF-PAEC cells displayed reduced TEER compared to PAEC (Figure 7). This is consistent with our data (Totani et al, unpublished) and previous studies ${ }^{11}$ that document alterations in the barrier function of CF endothelial cells. These findings support the hypothesis that a dysfunctional endothelium can contribute 
to sustain CF inflammation, in addition of representing a cardiovascular risk factor. Indeed, microvascular dysfunction has been reported in CF patients, ${ }^{27}$ which in association with inflammation, oxidant stress, and extension of the life expectancy, may increase the cardiovascular risk for $\mathrm{CF}$ patients. To study these phenomena, an adequate supply of endothelial cells from CF patients, preferably representative of the different class of CFTR mutations, is indispensable. These cells are difficult to obtain, since they can only derive from explanted organs. Moreover, primary endothelial cells have a very limited life span, in vitro, limiting their experimental usability. Thus, a collection of immortalized CF endothelial cells with different genotype may represent, similarly to $\mathrm{CF}$ respiratory cells lines, ${ }^{28}$ a valuable tool for studies on mechanisms and processes of pathogenetic relevance in CF.

As a conclusion, we report here a simple, fast, and highly efficient and reproducible methodology to obtain virtually pure EC populations from surgical specimens. This methodology that can be used to isolate EC from other sources, enabled us to obtain for the first time pure EC from CF patients. These cells can be now used for in vitro studies aimed to a better understanding of $\mathrm{CF}$ pathogenetic mechanisms as well as to drug discovery and personalized medicine.

\section{ACKNOWLEDGMENTS}

This work was fully supported by the Italian Cystic Fibrosis Foundation to MR (FFC\#17/2012, FFC\#19/2013, FFC\#23/2014). We thank all the patients and health operators for their invaluable support. We thank Laura Pierdomenico and Giuseppina Bologna for support with cytometric analyses. We also thank Valentina Crocetta, Arianna Pompilio, and Giovanni Di Bonaventura as well as Emanuela Caci and Luis Galietta for their precious advice and support with the anti-microbial strategy.

\section{DISCLOSURE/CONFLICT OF INTEREST}

The authors declare no conflict of interest.

1. Muller WA. Leukocyte-endothelial cell interactions in the inflammatory response. Lab Invest 2002;82:521-533.

2. Sumpio BE, Riley JT, Dardik A. Cells in focus: endothelial cell. Int J Biochem Cell Biol 2002;34:1508-1512.

3. Deanfield JE, Halcox JP, Rabelink TJ. Endothelial function and dysfunction: testing and clinical relevance. Circulation 2007;115: $1285-1295$.

4. Riordan JR, Rommens JM, Kerem B, et al. Identification of the cystic fibrosis gene: cloning and characterization of complementary DNA. Science 1989;245:1066-1073.

5. Stoltz DA, Meyerholz DK, Welsh MJ. Origins of cystic fibrosis lung disease. N Engl J Med 2015;372:1574-1575.

6. Mattoscio D, Evangelista V, De Cristofaro R, et al. Cystic fibrosis transmembrane conductance regulator (CFTR) expression in human platelets: impact on mediators and mechanisms of the inflammatory response. FASEB J 2010;24:3970-3980.
7. Del Porto P, Cifani N, Guarnieri S, et al. Dysfunctional CFTR alters the bactericidal activity of human macrophages against Pseudomonas aeruginosa. PLoS ONE 2011;6:e19970.

8. Sorio C, Buffelli M, Angiari C, et al. Defective CFTR expression and function are detectable in blood monocytes: development of a new blood test for cystic fibrosis. PLoS ONE 2011;6:e22212.

9. Noe J, Petrusca D, Rush N, et al. CFTR regulation of intracellular pH and ceramides is required for lung endothelial cell apoptosis. Am J Respir Cell Mol Biol 2009;41:314-323.

10. Romano $M$, Collura $M$, Lapichino $L$, et al. Endothelial perturbation in cystic fibrosis. Thromb Haemost 2001;86:1363-1367.

11. Brown MB, Hunt $\mathrm{WR}$, Noe JE, et al. Loss of cystic fibrosis transmembrane conductance regulator impairs lung endothelial cell barrier function and increases susceptibility to microvascular damage from cigarette smoke. Pulm Circ 2014;4:260-268.

12. Comhair SAA, Xu W, Mavrakis $L$, et al. Human primary lung endothelial cells in culture. Am J Respir Cell Mol Biol 2012;46:723-730.

13. Lachmann R, Lanuti P, Miscia S. OMIP-011: characterization of circulating endothelial cells (CECs) in peripheral blood. Cytometry 2012;81:549-551.

14. Kubota Y. Role of laminin and basement membrane in the morphological differentiation of human endothelial cells into capillary-like structures. J Cell Biol 1988;107:1589-1598.

15. Stewart SA, Dykxhoorn DM, Palliser D, et al. Lentivirus-delivered stable gene silencing by RNAi in primary cells. RNA 2003;4:493-501.

16. Zhao JJ, Gjoerup OV, Subramanian RR, et al. Human mammary epithelial cell transformation through the activation of phosphatidilinositol 3-kinase. Cancer Cell 2003;3:483-495.

17. Gimbrone MA, Cotran RS, Folkman J. Human vascular endothelial cells in culture. Growth and DNA synthesis. J Cell Biol 1974;60:673-684.

18. Bardin N. Identification of CD146 as a component of the endothelial junction involved in the control of cell-cell cohesion. Blood 2001;98: 3677-3684.

19. Lanuti $P$, Rotta G, Almici $C$, et al. Endothelial progenitor cells, defined by the simultaneous surface expression of VEGFR2 and CD133, are not detectable in healthy peripheral and cord blood. Cytometry 2016;89: 259-270.

20. McColley SA, Stellmach V, Boas SR, et al. Serum vascular endothelial growth factor is elevated in cystic fibrosis and decreases with treatment of acute pulmonary exacerbation. Am J Respir Crit Care Med 2000;161:1877-1880.

21. Rao S. New insights into pulmonary inflammation in cystic fibrosis. Arch Dis Child 2006:91:786-788.

22. Poore S, Berry B, Eidson D, et al. Evidence of vascular endothelial dysfunction in young patients with cystic fibrosis. Chest J 2013;143: 939-945.

23. Lyczak JB, Cannon CL, Pier GB. Lung infections associated with cystic fibrosis. Clin Microbiol Rev 2002;15:194-222.

24. Lanuti $P$, Serafini F, Pierdomenico L, et al. Human mesenchymal stem cells reendothelialize porcine heart valve scaffolds: novel perspectives in heart valve tissue engineering. Biores Open Access 2015;4:288-297.

25. Aguilera V, Briceño $\mathrm{L}$, Contreras $\mathrm{H}$, et al. Endothelium trans differentiated from Wharton's jelly mesenchymal cells promote tissue regeneration: potential role of soluble pro-angiogenic factors. PLOS ONE 2014;9:e111025.

26. De Francesco F, Tirino V, Desiderio V, et al. Human CD34/CD90 ASCs are capable of growing as sphere clusters, producing high levels of VEGF and forming capillaries. PLoS ONE 2009;4:e6537.

27. Rodriguez-Miguelez P, Thomas J, Seigler N, et al. 2016Evidence of microvascular dysfunction in patients with cystic fibrosis. Am J Physiol Heart Circ Physiol 2016;310:H1479-H1485.

28. Gruenert DC, Willems M, Cassiman JJ, et al. Established cell lines used in cystic fibrosis research. J Cyst Fibros 2004;3:191-196. 